\title{
Coup de bélier dû à une fermeture instantanée dans une conduite à caractéristiques multiples
}

\section{Water hammer caused by instantaneous closure in a multiple characteristic pipe}

\author{
PAR I. ESCANDE \\ MEMBRE DE L'INSTITUT, \\ DHECTEUR DE L'ÉCOLE NATIONALE SUPRIEURE D'ÉLECTROTECHNOUE ET D'HYDHALQUE DE TOULOUSE
}

\begin{abstract}
Cette étude du maximum d'amplitude des surpressions ef depressions consécutives a une fermeture complete instantanée, dans le cas d'une conduite $\dot{a}$ caracteristiques multiples, met en évidence des valeurs supérientes à celles que conduirait a envisager, swivant un raisonnement pourtant séduisant a priori, la considiration du seul troncon, mettant en jeu la bitesse et la célérité les plus élevées.
\end{abstract}

This investigation of the maximum amplitude of overpressures and depressions resulting from complete instantaneous closure in a multiple characteristic pipe, prodaces higher values than those we would expect to get by considering a single section of pipe and introducing maximum flow speed and wave speed, which wonld however seem to be the most attractive procedure on first sight.
Considérons une conduite horizontale de section $\mathrm{S}$, de diamètre $\mathrm{D}$, d'épaisseur $e$, constants sur toute sa longueur L, parcourue par un débit de régime permanent $q_{0}$ à la vitesse $V_{0}$ telle que :

$$
q_{0}=V_{0} \mathrm{~S}
$$

On sait qu'une fermeture instantanée complète de l'extrémité aval de cette conduite provoque, en l'absence de poches d'air ou de phénomènes de cavitations, un régime de surpressions et de dépressions alternées d'amplitudes égales à :

$$
\xi^{\prime}=\frac{a \mathrm{~V}_{0}}{g}, \xi^{\prime \prime}=-\frac{a \mathrm{~V}_{0}}{g}
$$

a désignant la vitesse de propagation des ondes dans la conduite, de période :

$$
\mathrm{T}=\frac{4 \mathrm{~L}}{a}=2 \theta
$$

A représentant la durée d'un aller et retour d'onde d'un bout à l'autre de la conduite.

Pour qu'il ne se produise pas de cavitations, i] faut évidemment que l'on ait :

$$
\frac{a \mathrm{~V}_{0}}{g}<y_{0}+y_{a t}
$$

$y_{0}$ désignant la pression statique sur l'axe de la 
conduite et $y_{a t}$ la pression atmosphérique, toutes deux exprimées en hauteur d'eau.

Le problème se présente de façon moins simple pour les conduites à caractéristiques multiples des usines de hautes chutes dont l'épaisseur augmente constamment de l'amont vers l'aval et dont la section elle-même peut correspondre à plusieurs diamètres différents, allant en diminuant de l'amont vers l'aval dans le cas des conduites télescopiques.

Dans tous les cas pratiques, la pente :

$$
\frac{a}{g \mathrm{~S}}
$$

des droites caractéristiques de la méthode graphique de Schnyder-Bergeron augmente de l'amont vers l'aval, du fait de la croissance de $a$ avec $e$ ou de la décroissance de $\mathrm{S}$.

On sait, d'autre part, qu'une fermeture instantanée de l'extrémité aval d'une conduite à caractéristiques multiples engendre une surpres- sion supérieure à celle que donne l'expression :

$$
\frac{a_{m}}{g} \frac{V_{0 m}}{g}
$$

$a_{m}$ étant la vitesse moyenne de propagation des ondes le long de la conduite et $V_{0 m}$ la vitesse pondérée équivalente à l'ensemble des vitesses mises en jeu dans les divers troncons de la conduite, compte tenu des masses liquides correspondantes; pour une conduite à diamètre constant, on a évidemment : $V_{0 m}=V_{0}$.

Dans le présent travail, nous exposons les résultats que nous avons obtenus en étudiant le maximum d'amplitude des surpressions et dépressions consécutives à une fermeture complète instantanée dans le cas d'une conduite à caractéristiques multiples.

Cette étude met en évidence des valeurs supérieures à celles que conduirait à envisager, suivant un raisonnement pourtant séduisant a priori, la considération du seul troncon aval, mettant en jeu la vitesse et la célérité les plus ćlevées.
Considérons, en effet, une conduite télescopique dans laquelle le troncon aval de longueur $L_{1}$ correspond à la plus petite section $\mathrm{S}_{1}$ et à la plus forte vitesse de propagation des ondes $a_{1}$, la vitesse de régime permanent $V_{01}$ étant donc supérieure à la valeur qu'elle possède dans les tronçons placés plus en amont.

Une fermeture instantanée de l'extrémité aval A engendre immédiatement en ce point une surpression :

$$
\xi_{1}^{\prime}=\frac{a_{1} \mathrm{~V}_{01}}{g}=\frac{a_{1} q_{0}}{g \mathrm{~S}}
$$

qui subsiste intégralement jusqu'à l'instant :

$$
\theta_{1}=\frac{2 \mathrm{~L}_{1}}{a_{1}}
$$

où le retour en A de l'onde réfléchie au changement de caractéristique réduit quelque peu la surpression initiale. On a évidemment :

$$
\xi_{1}>\frac{a_{m} \mathrm{~V}_{0 m}}{g}
$$

du fait que, par hypothèse, on vérifie les inégalités :

$$
a_{1}>a_{m} \quad \text { et } \quad V_{01}>V_{0 m}
$$

Le raisonnement auquel nous avons fait précédemment allusion serait alors le suivant:

Si la portion amont de longueur $\left(I_{-}-L_{1}\right)$ de la conduite avait les mêmes caractéristiques que le tronçon aval $\mathbf{L}_{1}$, $\xi_{1}$ donné par l'expression (3) représenterait l'amplitude maximum des surpressions et dépressions consécutives.

Le fait, qu'en réalité, dans cette portion amont de la conduite de longueur $\left(L-L_{1}\right)$ les vitesses $V_{0}$ et les célérités $a$ sont respectivement inférieures aux valeurs $V_{01}$ et $a_{1}$, semblerait devoir inciter à penser que les surpressions et dépressions engendrées dans la conduite considérée ne pourraient avoir que des amplitudes inférieures ou au plus égales à celle de $\xi_{1}^{\prime}$.

Il n'en est rien et, par le jeu des réflexions successives, les amplitudes de la surpression et de la dépression maxima peuvent dépasser $\xi_{1}^{\prime}$, comme nous allons le montrer. 


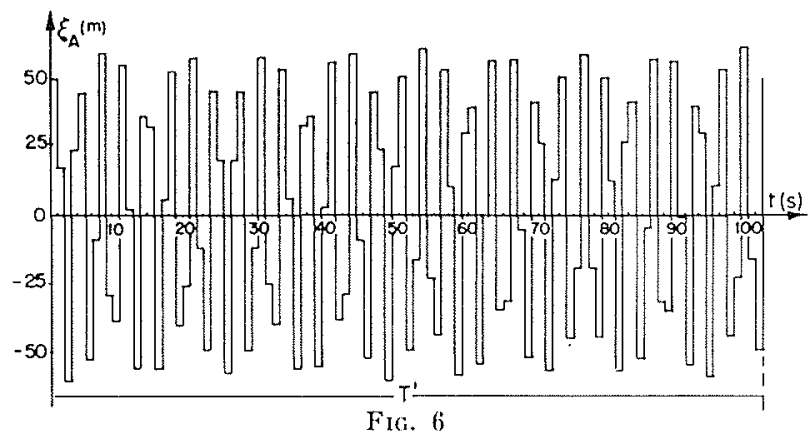

En ce qui concerne la dépression maximum maximorum, elle est réaliscée d'emblée pendant la $3^{\circ}$ seconde, avec la même amplitude de $61 \mathrm{~m}$.

La surpression et la dépression maxima ont donc une amplitude commune de $61 \mathrm{~m}$ supérieure de $22 \%$ à $\xi_{1}=50 \mathrm{~m}$, ce qui démontre bien le résultat que nous avons énoncé.

En pratique, la dépression maximum intervenant à la $3^{\mathrm{e}}$ seconde, l'onde n'aura guère subi d'amortissement appréciable et la valeur théorique de $61 \mathrm{~m}$ sera bien effectivement atteinte : il n'en sera probablement pas de même de la surpression maximum théorique qui ne se produit

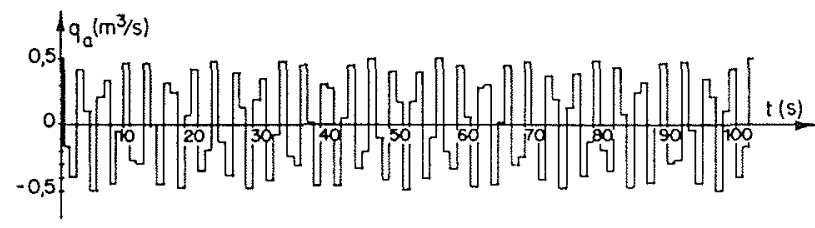

Fig. 7

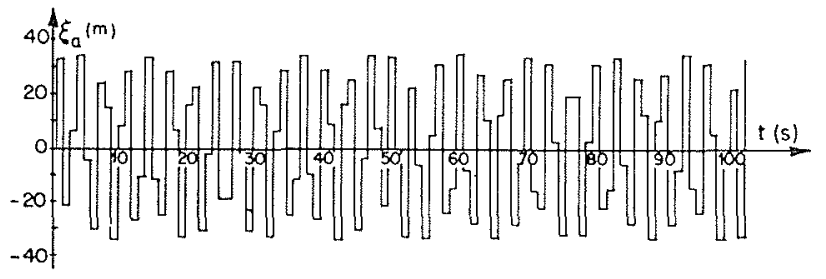

FIa. 8

qu'à la $54^{\circ}$ seconde, mais, par contre, la valeur de $59 \mathrm{~m}$, relative à la $8^{\circ}$ seconde, aura toutes chances d'être sensiblement réaliscée.

Les figures 7 et 8 reproduisent les courbes donnant respectivement en fonction du temps, les débits, d'une part, et les surpressions, d'autre part, au point $a$.

Enfin, la figure 9 est relative au débit en B : on observe l'amplitude des variations qui atteint $0,84 \mathrm{~m} \% / \mathrm{s}$, valeur très supérieure à celle de $0,5 \mathrm{~m} \% / \mathrm{s}$ qui interviendrait dans le cas d'une conduite à caractéristique unique sur toute sa longueur.

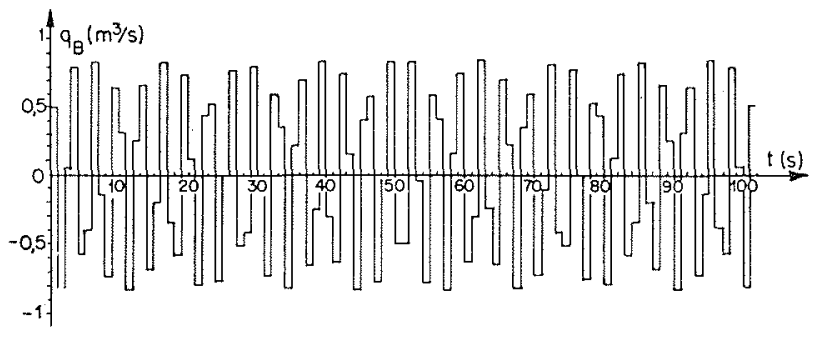

FiG. 9
Nous venons d'étudier, sur un cas particulier de conduite à caractéristiques multiples constituée de deux tronçons, la surpression produite par une fermeture instantanée, en supposant la pression statique $y_{0}$ assez grande pour qu'il n'y ait pas de cavitations.

Nous reprenons l'étude du même problème, avec les mèmes données numériques, mais en supposant maintenant la pression statique $y_{0}$ égale à $40 \mathrm{~m}$ et la pression atmosphérique $y_{a t}$ égale à $10 \mathrm{~m}$.

Dans ces conditions, on a toujours :

$$
\xi_{1}^{\prime}=\frac{a_{1} V_{01}}{g}=50 \mathrm{~m}
$$

de telle sorte que :

$$
\xi_{1}^{\prime}=y_{0}+y_{a t}
$$

Dans le cas d'une conduite à caractéristique unique sur toute sa longueur correspondant aux valeurs $a_{1}$ et $V_{01}$, on se trouverait donc exactement placé à la limite voulue pour que n'apparaissent pas les cavilations.

Par contre, avec la conduite composée de deux tronçons, dès le premier retour d'onde, pendant la $3^{\circ}$ seconde, comme le montre la construction graphique de la figure 10 , une cavité se forme à l'extrémité aval de la conduite, en $\mathrm{A}$ : de ce fait, dès la 5 " seconde, il existe en A une surpression égale à $72 \mathrm{~m}$ et done très supérieure à la surpression maximum de $61 \mathrm{~m}$ constatée en l'absence de cavitations.

Nous avons poursuivi, comme le montrent les figures successives 11,12 et 13 , les diverses étapes de la construction graphique pour voir si le maximum de $72 \mathrm{~m}$, constaté à la $3 "$ seconde, ne pouvait pas être dépassé dans la suite. 

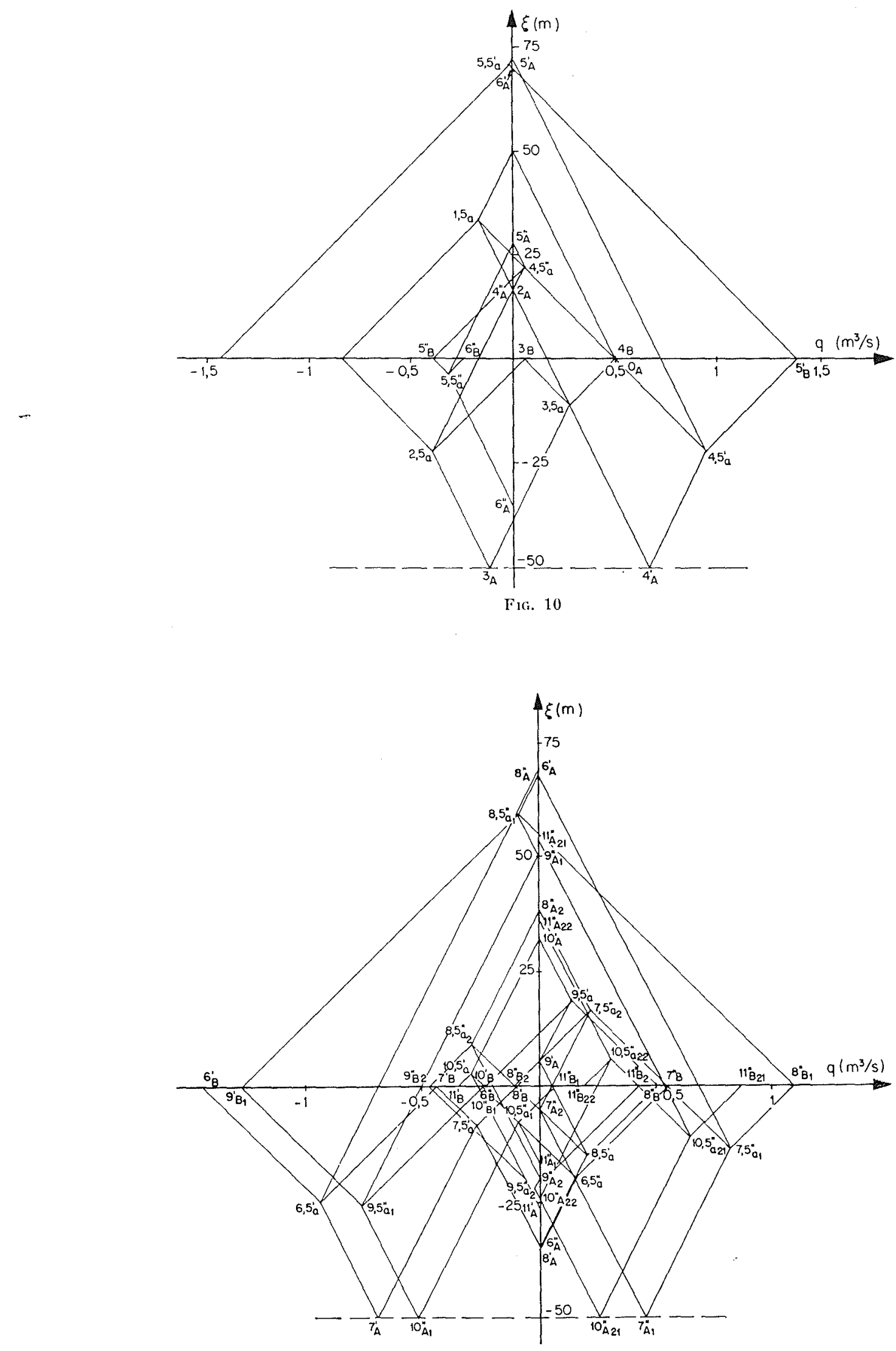

Fig. 11 

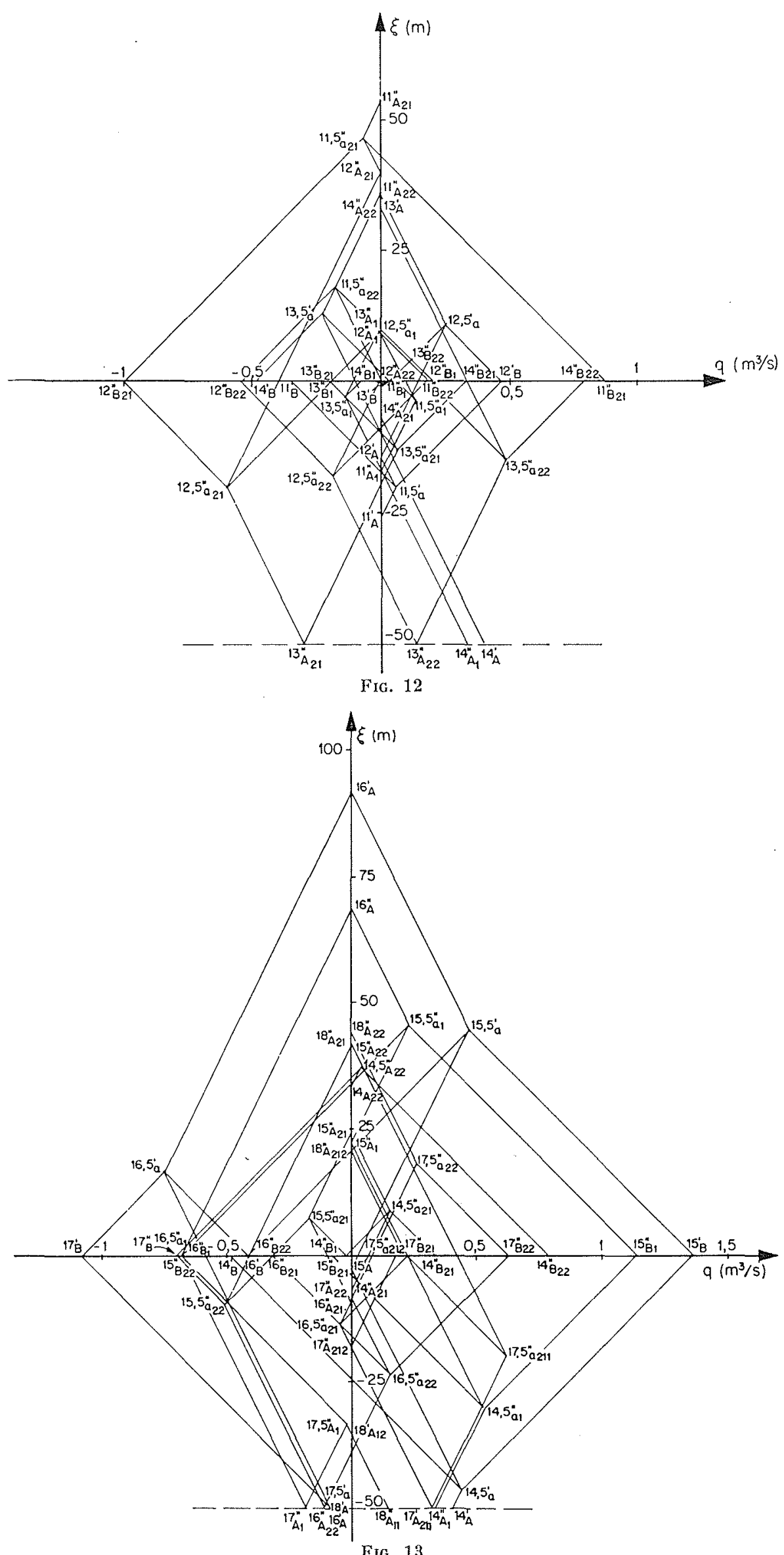

FIG. 13 
En fait, comme on le voit sur la figure 13 , pendant une fraction de la $16^{\circ}$ seconde, la surpression atteint $92 \mathrm{~m}$.

Etant donné la complication de la construction graphique résultant du fractionnement de chaque intervalle de temps, par le jeu des cavités intermittentes engendrées en A, nous n'avons pas poussé plus loin la construction graphique, de telle sorte que nous ne savons si cette valeur de $92 \mathrm{~m}$ ne serait pas elle-même dépassée par la suite.

Quoiqu'il en soit, il suffit de rapprocher les chiffres de $72 \mathrm{~m}$ et de $92 \mathrm{~m}$ du maximum de $61 \mathrm{~m}$, que nous avions obtenu dans l'étude relative au cas où il n'y a pas de cavitations, pour pouvoir conclure.

La constitution de la conduite en deux troncons de caractéristiques différentes, du fait qu'elle entraine un phénomène de cavitation, qui n'existerait pas si la conduite était à caractéristique unique, majore dans des proportions beaucoup plus importantes qu'en l'absence de cavitations, la surpression maximum engendrée au distributeur.

\section{I S CUS SION}

president: M. FEH M. le Président rend hommage à la clarté et à la
rapidité coutumières de l'exposé de M. Escande.
M. Escaxpe ajoute quelques mots a sa communication,
sur le fait de la fermeture instantanée, qui n'intervient
jamais en exploitation normale, mais que l'on rencontre
malheureusement dans le cas d'expertise d'accident. Il
rappelle un accident, survenu a Saint-Etienne-Cantalés,
où une vanne de 2 m environ de diamétre s'était fermée.
d'après le calcul, en 0,16 s. Ce n'est pas instantané, mais
e'est tout de même très court.

M. le Président signale qu'il a eu occasion de voir le résumé d'une étude, datant de 1938, où l'on schématisait la conduite à caractéristiques multiples de manière plus complexe. D'une part, le diamètre de la conduite, d'autre part, la célérité des ondes, variaient linéairement entre l'extrémité de la conduite et le réservoir. Avec ces domnées, en cas de fermeture instantanéc, on trouvait des majorations de l'ordre de $150 \%$ par rapport à la valeur de $a \mathrm{~V}_{0} / g$ correspondant aux caractéristiques ì l'extrémité aval, alors que M. Escanof arrive senlement a $22 \%$ pour le cas qu'il a étudié. C'est dire qu'en cas de fermeture instantanée, les conduites réelles n'auraient sans doute pas beaucoup de chances de survivre; mais heureusement les phénomènes véritablement instantanés n'existent pas.

M. Escande signale que, en dehors de la fermeture instantanée, ces résultats se maintiennent d'une façon à peu près integrale dans ce qu'on appelle la fermeture brusque qui, dans le cas d'une conduite à caractéristique unique, est celle qui se fait en un temps inférieur à $2 \mathrm{~L} / a$ (cas à Saint-Etienne-Cantalès).

M. Escande signale encore un autre cas, l'usine du Lac Noir, où il y avait des phénomènes de surpression très élevés, bien que non rigoureusement instantanés.

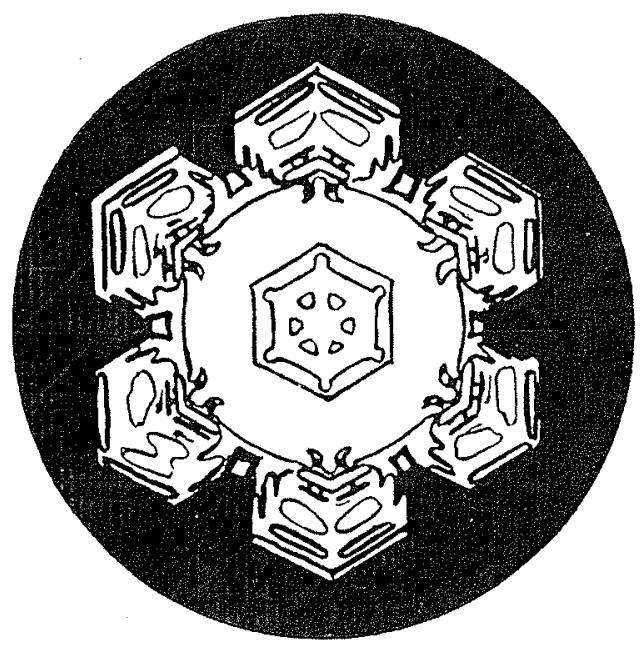

